\title{
Simulation of Polarized Positron Sources for Linear Colliders
}

\author{
A. Ushakov, A. Schälicke, S. Riemann
}

\author{
DESY, Zeuthen \\ PESP2010, \\ Workshop on Sources of Polarized Leptons and \\ High Brightness Electron Beams \\ Bonn, 24 September 2010
}




\section{Outline}

Motivation: Development reliable tool for positron source simulations

- Positron Source Components

- Simulation Tool PPS-Sim: Polarized Positron Source Simulation

- Simulation Results: Yield and Polarization

- AMD

- Li-Lens

- Quarter Wave Transformer

- Energy Deposition in Target (PEDD)

- Summary 


\section{ILC Positron Source Scheme (RDR Design)}

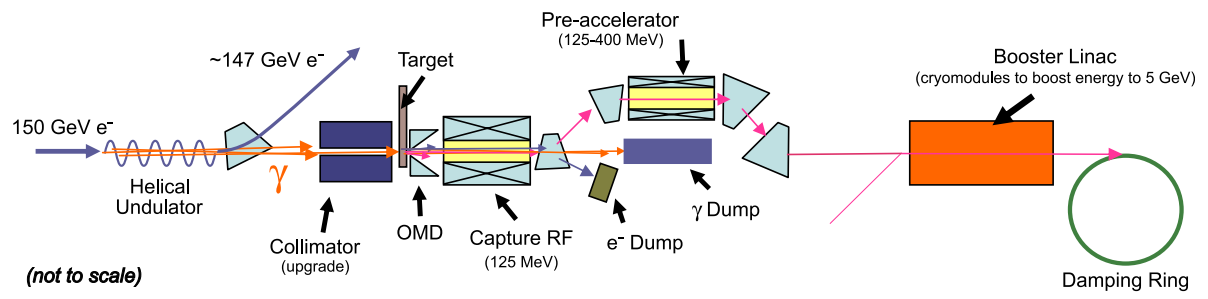

Aim: to simulate $\mathrm{e}^{+}$production, focusing/capturing and transport up to end of capture section (125 MeV) or up to DR 


\section{Positron of Source Components}

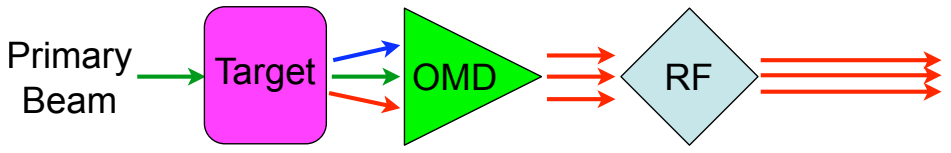

\section{Primary Beam}

- Undulator photons

- Electrons (conventional source)

- Input file (Compton photons, channeling radiation)

Target

- Solid wheel (Ti- or W-alloy)

- Liquid Lead
Optical Matching Device (OMD) and Accelerating Cavity (RF)

- Pulsed flux concentrator (AMD)

- Lithium lens

- Quarter-wave transformer (QWT)

- $1.3 \mathrm{GHz}$ cavity embedded into solenoid

\section{Damping Ring (DR)}

Photon Collimator (optionally) 


\section{Used Simulation Tools}

PPS-Sim is Geant4-based application for $\mathrm{e}^{+}$source modeling

- Electromagnetic and hadronic shower development in target

- Single particle tracking in electro-magnetic fields

- Polarization transfer in physics processes

- Spin tracking in electro-magnetic fields

- Powerful geometry package

- Visualisation of geometry model, particle trajectories and energy deposition

- Qt4-based Graphical User Interface (GUI)

- ROOT: analysis of results and input data (e.g. energy spectrum of primary beam) 


\section{Visualization Example}

Source Model with Liquid Lead Target and QWT

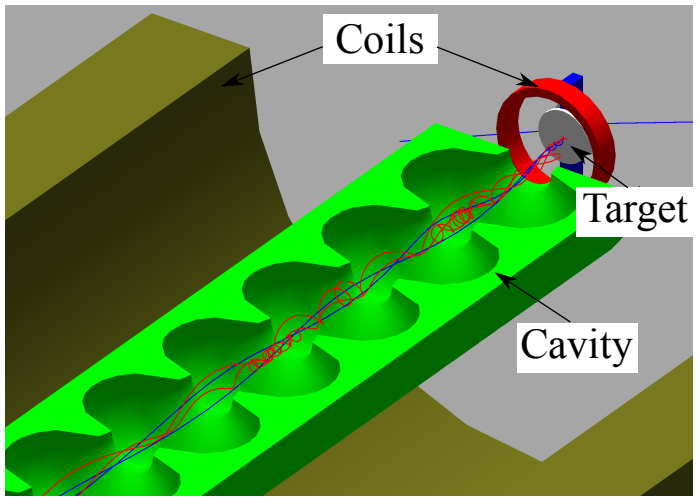




\section{PPS-Sim: Source Configuration}

Source can be configured via macro-commands (Geant4) or dialog "Preferences"

- Choice of source components

- Dimensions \& relative positions

- Beam, field parameters

- ...

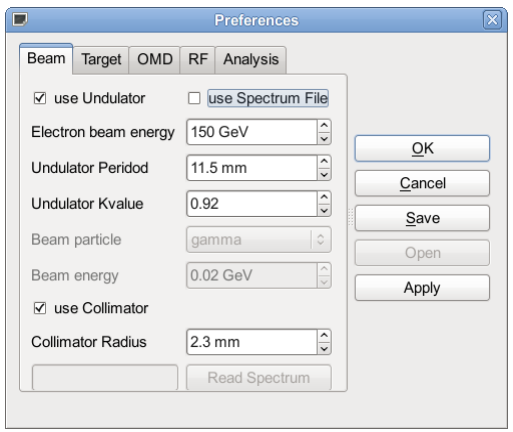

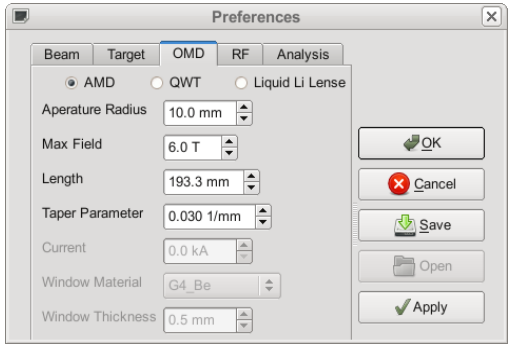




\section{PPS-Sim: Main Window and On-line Analysis}

\section{Main Window}

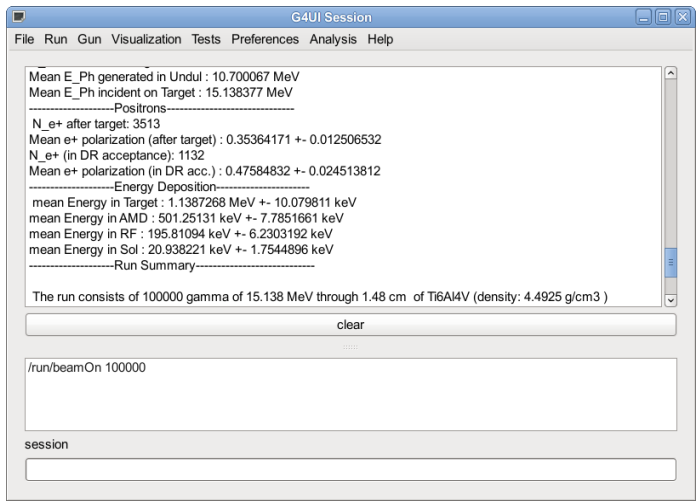

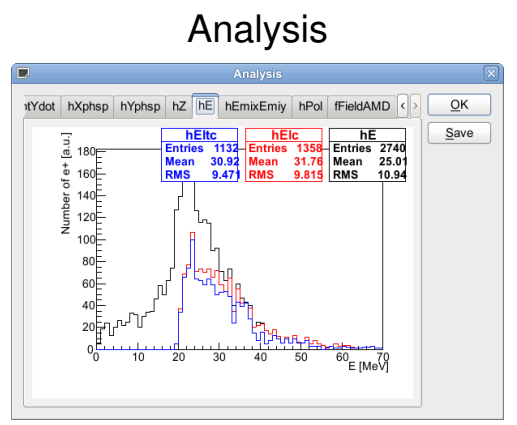




\title{
Photon Energy Distribution and Polarization
}

\author{
Helical Undulator: \\ $K=0.92$, Period $=11.5 \mathrm{~mm}$
}

Field on axis $=0.86 \mathrm{~T}$, Aperture $=5.85 \mathrm{~mm}$

$150 \mathrm{GeV}^{-}$Beam (RDR Design)

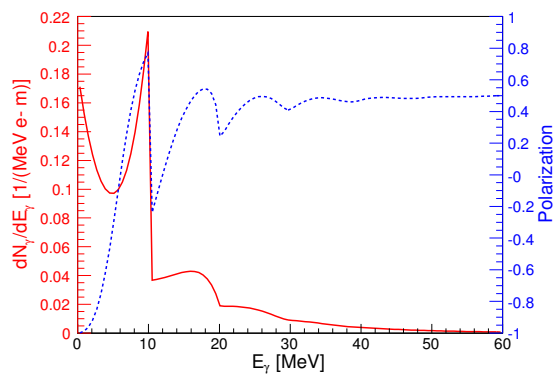

$E_{1} \simeq 10 \mathrm{MeV}$
$250 \mathrm{GeV}^{-}$Beam (SB2009)

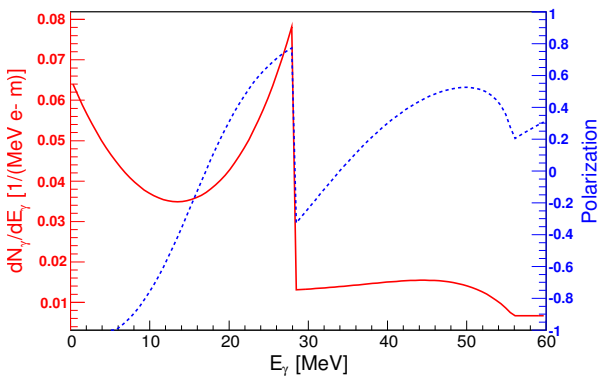

$E_{1} \simeq 28 \mathrm{MeV}$ 


\section{Flux Concentrator (AMD) Model}
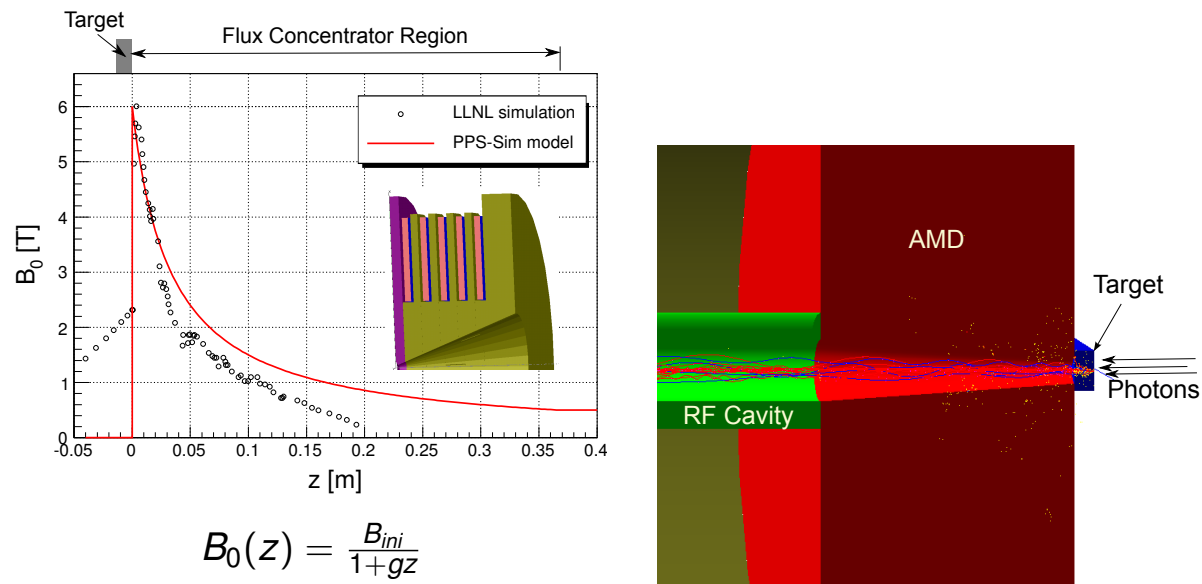

\begin{tabular}{|l|c|}
\hline Initial B-field, $\mathrm{T}$ & 6 \\
\hline End B-field, $\mathrm{T}$ & 0.5 \\
\hline Taper parameter $\mathrm{g}, \mathrm{m}^{-1}$ & 30 \\
\hline
\end{tabular}




\section{Yield and Polarization vs AMD Initial B-field}

Yield vs AMD Initial Field

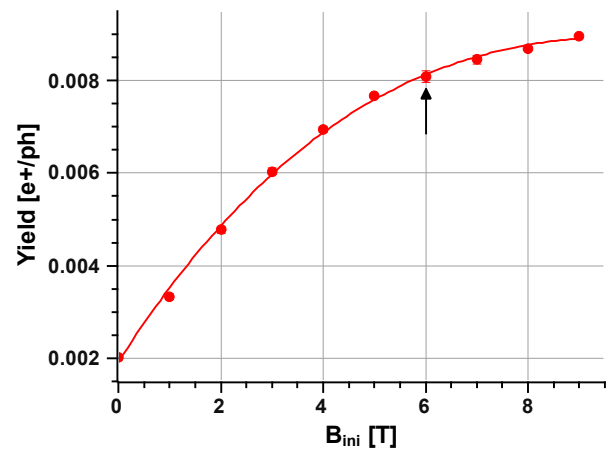

Polarization vs AMD Initial Field

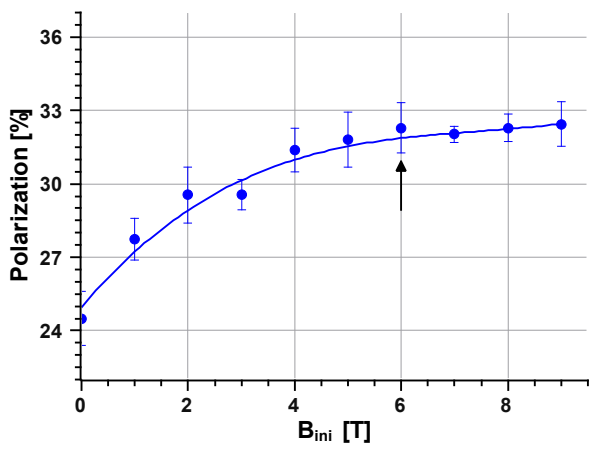




\section{Li-Lens Model}

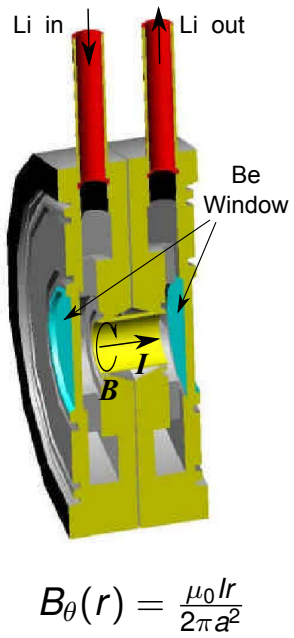

A. Mikhailichenko, Cornell University Report (2010) CBN 10-3

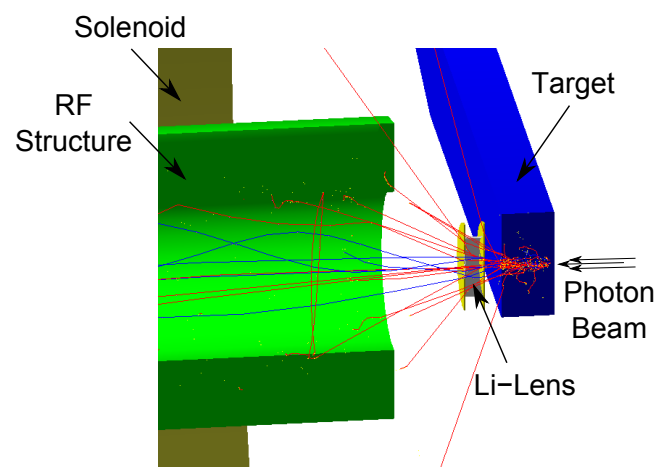

Issue:

Energy deposition in lens windows 


\section{Yield and Polarization vs Lens Current}
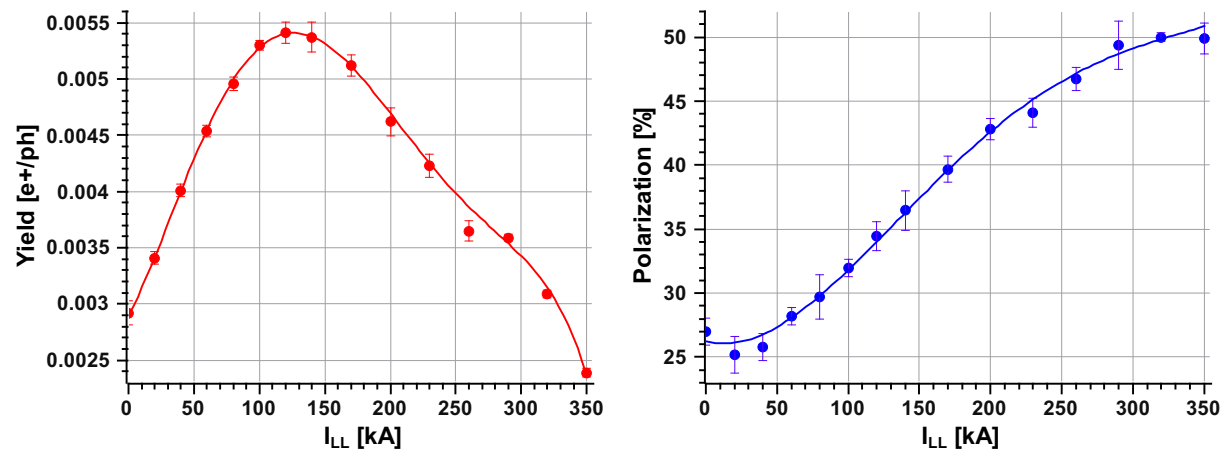

- Optimal lens current (for yield): $\simeq 120 \mathrm{kA}\left(0.52 \mathrm{kA} / \mathrm{mm}^{2}\right)$

- Higher lens field ("overfocussing") is better for polarization 


\section{QWT Model}

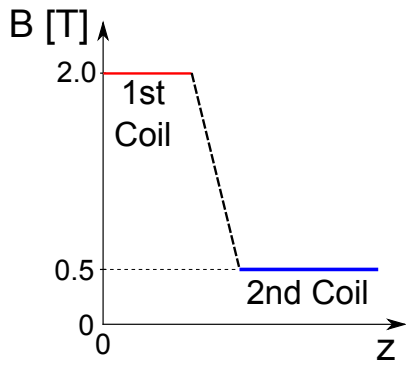

More realistic field distribution has been calculated and will be implemented in PPS-Sim

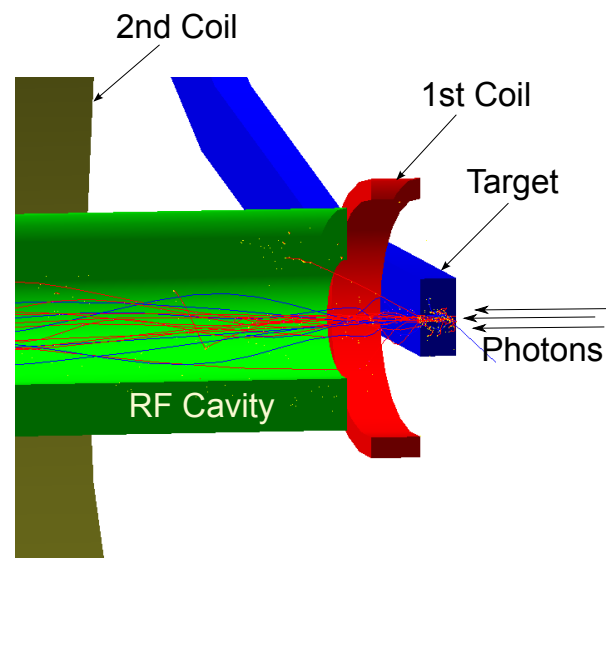




\section{Yield and Polarization vs Field of 1st Coil of QWT}
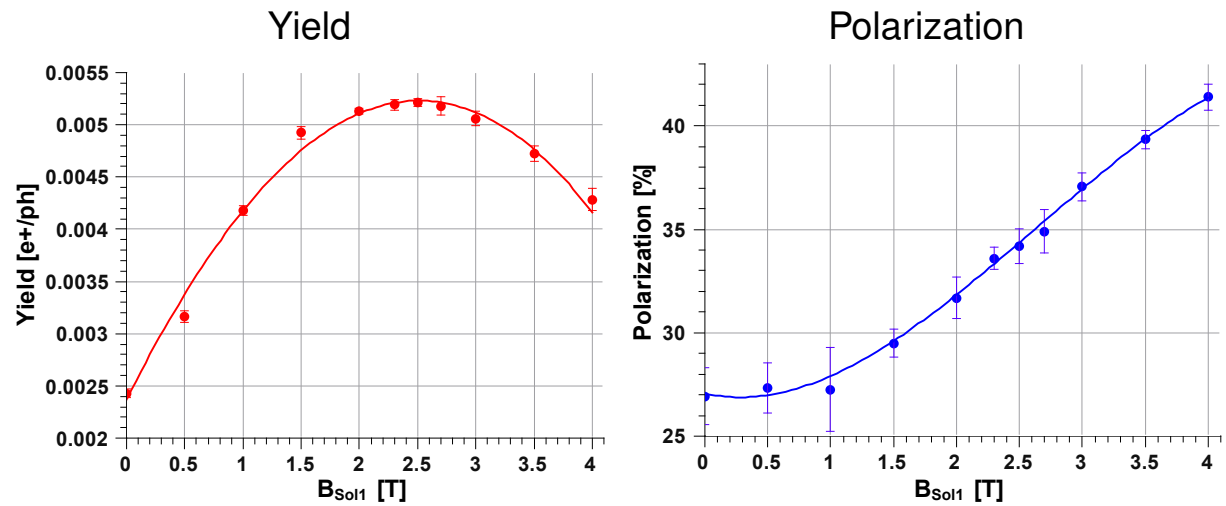

\begin{tabular}{|l|c|}
\hline Distance to Target, $\mathrm{mm}$ & 0 \\
\hline Distance to RF, $\mathrm{mm}$ & 10 \\
\hline \hline$B_{\text {Sol2 }}, \mathrm{T}$ & 0.5 \\
\hline
\end{tabular}




\section{Performance of AMD, Li-Lens and QWT}

\begin{tabular}{|c|c|c|c|}
\hline & AMD $(6 \mathrm{~T} \mapsto 0.5 \mathrm{~T})$ & Li-Lens & QWT (2.5 T) \\
\hline \hline Yield (after Target), $\mathrm{e}^{+} / \mathrm{ph}$ & \multicolumn{3}{|c|}{0.0226} \\
\hline "Captured" Yield, $\mathrm{e}^{+} / \mathrm{ph}$ & $8.1 \cdot 10^{-3}$ & $6.4 \cdot 10^{-3}$ & $5.2 \cdot 10^{-3}$ \\
\hline Capture Efficiency, \% & 35.8 & 28.3 & 23.1 \\
\hline Polarization, \% & 32.3 & 34.7 & 34.2 \\
\hline
\end{tabular}




\section{Comparison with other Simulation Programs (EGS+Elegant)}

\section{Capture Efficiency [\%]}

\begin{tabular}{|c||c|c|}
\hline OMD & ANL $^{1}$ & PPS-Sim \\
\hline \hline AMD, immersed target & $\sim 30$ & 35.8 \\
\hline Li-Lens $(50 \mathrm{MV} / \mathrm{m})$ & $\sim 29$ & 31.2 \\
\hline QWT $(1 \mathrm{~T}, 2 \mathrm{~cm})$ & $\sim 21$ & 18.5 \\
\hline 0.5 T Solenoid & $\sim 10$ & 10.7 \\
\hline
\end{tabular}

${ }^{1}$ Wanming Liu, Wei Gai et al., Positron Source Collaborating Meeting, Argonne, IL, USA, Sept. $17-19,2007$ 


\section{Photon Collimator for Positron Source at the End of Main Linac $(250 \mathrm{GeV})$}

Positron source at $250 \mathrm{GeV}$ (SB2009) provides much more (approx. 3 times) positrons that at $150 \mathrm{GeV}(\mathrm{RDR})$ for the same undulator length, but e+ polarization is about $22 \%$ only. To increase beam polarization the photon collimator have to be used.

Yield and Polarization vs Aperture Size of Photon Collimator

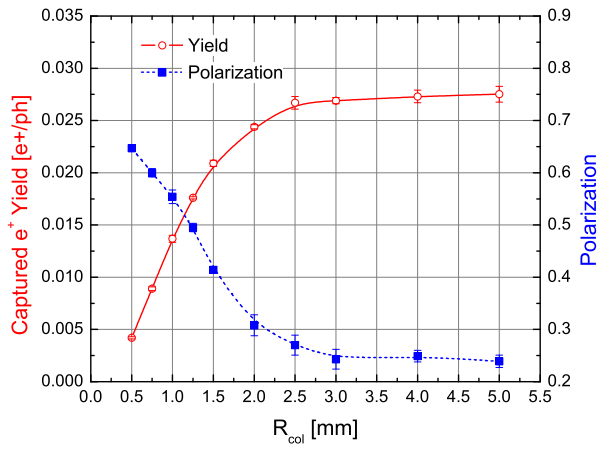

Required Undulator Photon Power and Deposited Power in Photon Collimator

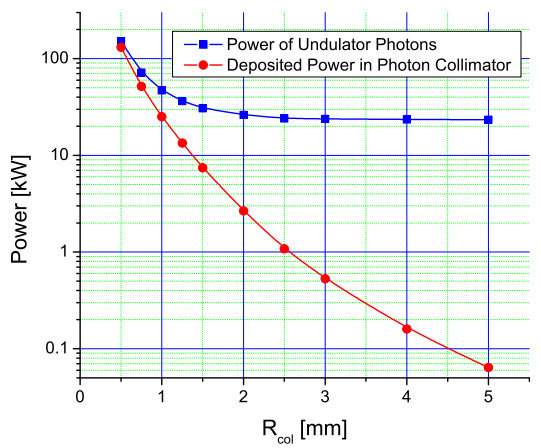




\section{Energy Deposition in Target. AMD Field from $6 \mathrm{~T}$ to $0.5 \mathrm{~T}$}

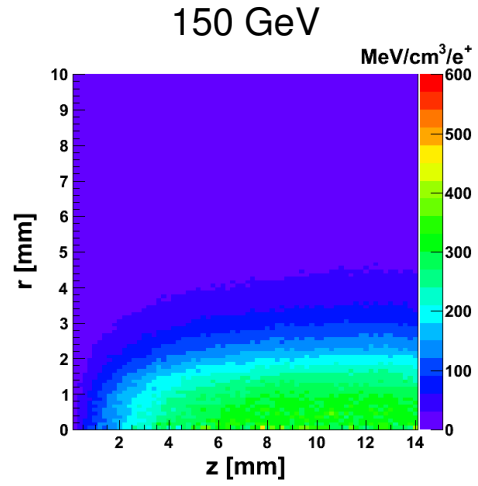

Total Energy: $92.7 \mathrm{MeV} / \mathrm{e}^{+}$ PEDD: $320.8 \mathrm{MeV} / \mathrm{e}^{+} / \mathrm{cm}^{3}$

$0.34 \mathrm{~J} / \mathrm{g} / \mathrm{bunch}$

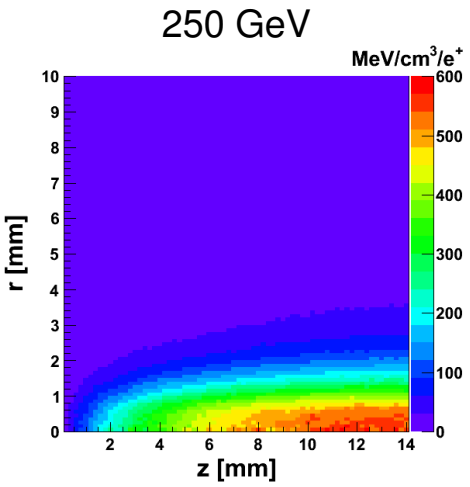

Total Energy: $60.4 \mathrm{MeV} / \mathrm{e}^{+}$ PEDD: $547.0 \mathrm{MeV} / \mathrm{e}^{+} / \mathrm{cm}^{3}$

$0.58 \mathrm{~J} / \mathrm{g} / \mathrm{bunch}$

PEDD - Peak Energy Deposition Density 


\section{PEDD: Comparison with other Programs}

\section{RDR undulator, AMD}

\section{PPS-Sim}

\begin{tabular}{|l|c|c|r|}
\hline & $150 \mathrm{GeV} \& 5 \mathrm{~T}$ & \multicolumn{2}{|c|}{$250 \mathrm{GeV} \& 6 \mathrm{~T}$} \\
\hline Total Deposited Energy & $100.4 \mathrm{MeV} / \mathrm{e}^{+}$ & $60.4 \mathrm{MeV} / \mathrm{e}^{+}$ & $60 \%$ \\
\hline PEDD & $348.8 \mathrm{MeV} / \mathrm{e}^{+} / \mathrm{cm}^{3}$ & $547.0 \mathrm{MeV} / \mathrm{e}^{+} / \mathrm{cm}^{3}$ & $157 \%$ \\
\hline
\end{tabular}

Elegant (Wei Gai, ALCPG, Albuquerque, 2009)

\begin{tabular}{|l|c|c|r|}
\hline & $150 \mathrm{GeV}$ & \multicolumn{2}{|c|}{$250 \mathrm{GeV}$} \\
\hline Total Deposited Energy & $101 \mathrm{MeV} / \mathrm{e}^{+}$ & $62.8 \mathrm{MeV} / \mathrm{e}^{+}$ & $62 \%$ \\
\hline PEDD & & & $160 \%$ \\
\hline
\end{tabular}




\section{Beam Transport Downstream $125 \mathrm{MeV}$}

ILC e+ PCAPA beamline

BMAD simulations for ILC polarized e+ beam transport downstream $125 \mathrm{MeV}$ have been started

PCAPA (Positron CAPture system A) is the beamline that separates the positrons from the electrons and photons
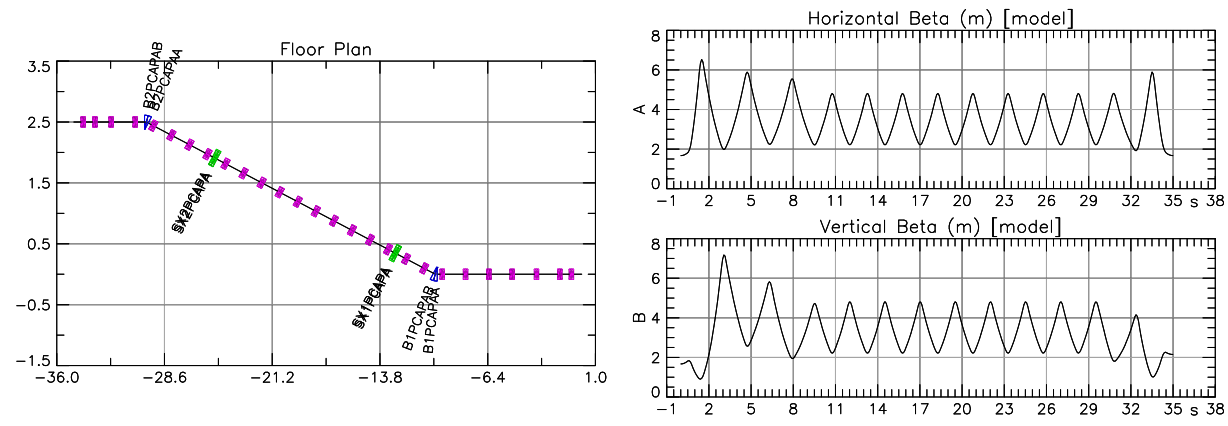


\section{Summary and Outlook}

- Geant4-based tool PPS-Sim for polarized positron source simulations has been developed

- A variety of e+ source options (different primary beams, targets, OMD's) are included

- Graphical User Interface simplifies usage

- OpenGL visualization of geometry provided

- PPS-Sim is open-source code and available for download: http://pps-sim.desy.de

Plans:

- Adding more realistic field (field maps) into PPS-Sim

- Automatically finding of optimal electrical field phase

- Beam tracking up to DR (including spin rotator) in PPS-Sim + Bmad 


\section{Backup Slides}




\section{Positron Yield}

Conventional Source with Liquid Lead Target and AMD

- $\mathrm{Pb}$ target, $3 \mathrm{~mm} \mathrm{BN}$ window

- Pencil-like $\mathrm{e}^{-}$beam

- AMD field: $6 \mathrm{~T}$ to $0.5 \mathrm{~T}$

- Optimized AMD taper parameter

- E-field: $14.5 \mathrm{MeV} / \mathrm{m}$

- DR acceptance: $0.09 \mathrm{~m}$ rad, $10 \mathrm{~mm}$ long. bunch size

"Captured" Positron Yield

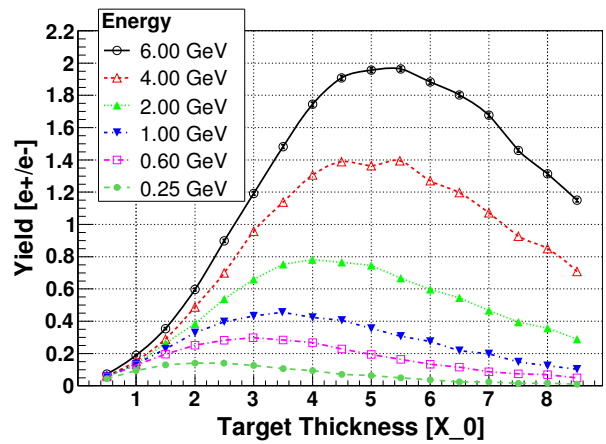




\section{PEDD for $6 \mathrm{GeV} \mathrm{e}^{-}$beam}

Conventional Source with Lead Target and AMD

\section{Energy Deposition in Target}

\begin{tabular}{|l|c|}
\hline $\mathrm{e}^{-}$beam energy & $\mathbf{6 ~ G e V}$ \\
\hline Beam size, $\sigma_{r}$ & $4.0 \mathrm{~mm}$ \\
\hline Target material & Lead \\
\hline Target density, $\rho$ & $11.35 \mathrm{~g} / \mathrm{cm}^{3}$ \\
\hline Target thickness & $5 X_{0}$ \\
\hline \hline Number of $\mathrm{e}^{+}$ & $3 \cdot 10^{10} \mathrm{per}^{\mathrm{bunch}}$ \\
\hline \hline Captured Yield & $0.84 \mathrm{e}^{+} / \mathrm{e}^{-}$ \\
\hline PEDD & $4.54 \mathrm{~J} / \mathrm{g} / \mathrm{bunch}$ \\
\hline
\end{tabular}

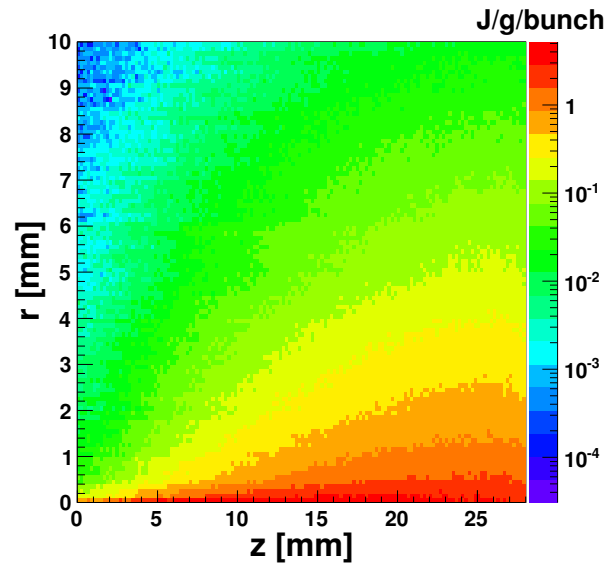

